\title{
Modelling of a dynamic torque calibration device and determination of model parameters
}

\author{
Leonard Klaus, Thomas Bruns, Michael Kobusch \\ Physikalisch-Technische Bundesanstalt (PTB), Bundesallee 100, 38116 Braunschweig, Germany
}

\section{ABSTRACT}

For the dynamic calibration of torque transducers, a dedicated calibration device has been developed. This paper is focused on the model of the calibration device and the methods for the determination of its model parameters. The modelling is required for the identification of the corresponding model parameters of the torque transducer under test. These parameters describe the transducer's dynamic behaviour. Measurement methods and auxiliary devices for the determination of mass moment of inertia and torsional stiffness are explained. This research is part of the EMRP JRP IND09 - "Traceable Dynamic Measurement of Mechanical Quantities".

\section{Section: RESEARCH PAPER}

Keywords: Dynamic torque calibration, mass moment of inertia, torsional stiffness, sinusoidal excitation, modelling of mechanical systems.

Citation: Leonard Klaus, Thomas Bruns, Michael Kobusch, Modelling of a dynamic torque calibration device and determination of model parameters, Acta IMEKO, vol. 03, no. 2, article 5, June 2014, identifier: IMEKO-ACTA-03 (2014)-02-05

Editor: Paolo Carbone, University of Perugia

Received February 15 $5^{\text {th }}, 2013$; In final form August $1^{\text {st }}, 2013$; Published June 2014

Copyright: (C) 2014 IMEKO. This is an open-access article distributed under the terms of the Creative Commons Attribution 3.0 License, which permits unrestricted use, distribution, and reproduction in any medium, provided the original author and source are credited

Funding: The research leading to these results has received funding from the European Union on the basis of Decision No $912 / 2009 / E C$.

Corresponding author: Leonard Klaus, leonard.klaus@ptb.de

\section{INTRODUCTION}

Dynamic torque applications exist in many fields of industry; two examples are engine test stands and power torque tools. Efficiency measurements of combustion engines and electric drives are carried out by means of rotational speed and dynamic torque measurements in engine test stands. The torque output of these drives may be significantly dynamic with a frequency content up to the kilohertz range.

From experience with force transducers - which have a related mechanical design, measurement principle and installation situation - it is known that transducers may have a dynamic behaviour which can influence the measuring results.

At present, static torque calibrations can be carried out in a wide range and with high precision, but there are no facilities and standards to determine the dynamic behaviour of torque transducers. For this reason, a method for primary dynamic torque calibration was developed and a proof-of-principle measuring device was designed and manufactured at PTB.

\section{MEASURING DEVICE}

The dynamic torque measuring device enables dynamic torque excitations in a frequency range of up to $1 \mathrm{kHz}$ with up to $20 \mathrm{~N} \cdot \mathrm{m}$ sinusoidal torque.
The measurement principle is in analogy to the periodic force excitation based on Newton's second law [1]. With the mass moment of inertia $J$ and the time-varying angular acceleration $\ddot{\varphi}(t)$, the torque $M(t)$ is given by

$M(t)=\ddot{\varphi}(t) \cdot J$

Figure 1 shows the components of the calibration device and the corresponding mechanical spring-mass-damper model. The transducer under test (DUT) is mounted via two coupling elements to a rotational exciter. The coupling elements are composed of an upper and a lower half connected by a steel diaphragm to reduce parasitic bending moments acting on the transducer. At the same time, this mechanical design offers a high torsional stiffness. Interchangeable collets enable the mounting of transducers with shaft ends of various diameters in the measuring device. At the top of the set-up, the transducer under test carries a known mass moment of inertia (MMOI) $J$, which is composed of components for an angular acceleration measurement set-up and a radial air bearing. The air bearing reduces the axial load as well as bending moments acting on the transducer under test in conjunction with low friction operation. 
The primary measurement of the angular acceleration is carried out by means of a radial grating disk and a laserDoppler interferometer [1].

\section{MODEL}

The measurement principle of the vast majority of torque transducers is based on strain gauges. This principle is used for force transducers as well, so the implementation of a model for torque transducers was realised based on the experience with force transducers [2]. Strain gauge transducers have a structural design, in which the sensing elements are applied on a structural part of high compliance. This part can be described as a torsional spring $\left(c_{\mathrm{T}}\right)$ for torque transducers. The remaining structural parts of the transducer can be assumed to be rigid and will be allocated to the mass moment of inertia element above $\left(J_{\mathrm{H}}\right)$ or below $\left(J_{\mathrm{B}}\right)$ the torsional spring, respectively. In parallel to the torsional spring, the model assumes a damping element. The behaviour of this system is supposed to be linear and time invariant (LTI).

The distribution of the head and base MMOI of the transducer under test $\left(J_{\mathrm{H}}\right.$ and $\left.J_{\mathrm{B}}\right)$ depends on the mechanical design of the transducer.

Because of the fact that torque transducers are always coupled to the mechanical environment at both ends, different coupled elements of different MMOI, stiffness and damping may have influence on the frequency response of the system, which affects the frequency-dependent output signal of the transducer. To be able to identify the model parameters of the transducer, it is necessary to include the entire measurement set-up in the model. Again, a linear and time invariant approach is applied.

As the coupling elements cannot be assumed to be totally rigid to torsional loads, they are represented in the model of the measuring device by two torsional spring and damper elements $\left(c_{\mathrm{M}}, d_{\mathrm{M}} ; c_{\mathrm{E}}, d_{\mathrm{E}}\right)$ coupled to mass moments of inertia at each end. The MMOI values of the rigid connections of the

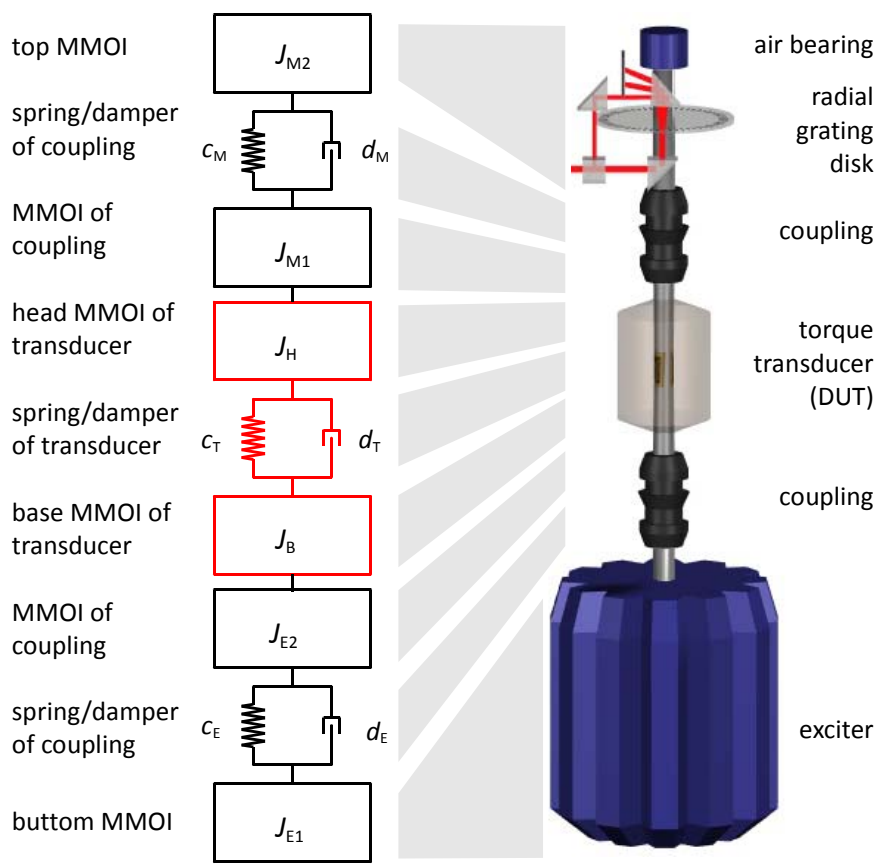

Figure 1. Measuring device and its model. couplings and the transducer are split and accordingly allocated to the transducer and to the measuring device (e.g. $J_{E 2}$ and $J_{B}$ in Figure 1) in order to derive the model parameters of the transducer.

The mass moment of inertia $J_{M 2}$ includes all components of the measuring device on top of the upper half of the coupling. $J_{M 1}$ includes the lower part of the coupling, as well as adapters for the mounting of the transducer; the same applies to $J_{E 2}$ but in the opposite direction.

Only if the model parameters of the measuring device are known, the determination of the transducer's model parameters from measurement data will be possible. Thus, measurement techniques for the determination of these parameters have to be developed.

\section{MASS MOMENT OF INERTIA}

The mass moment of inertia that generates the dynamic torque acting on the transducer, when applied to an angular acceleration, needs to be determined with high precision.

For this purpose, a dedicated measuring device for the mass moment of inertia has been developed (see Figures 2,3). Its measurement method is based on the principle of a physical pendulum. For small angles of pendulum excitation, the nonlinearity of the pendulum can be neglected - though this effect has to be taken into account for the measurement uncertainty evaluation. The frequency $\omega$ of the pendulum's swing depends on its mass moment of inertia $J$ and the restoring torque given by the mass of the pendulum $m$, the distance of the centre of mass from the axis of rotation $l$ and the gravitational acceleration $g$.

$J=\frac{m \cdot g \cdot l}{\omega^{2}}$.

To minimize frictional losses, the pendulum set-up uses an air bearing [3]. The DUT is mounted on the axis of rotation. The mass moment of inertia of the DUT is derived from the change of the pendulum's swing frequency. For the measurement of the angular excitation, an optical angle encoder is used. Its grating disk has an angular pitch of $0.04^{\circ}$. This

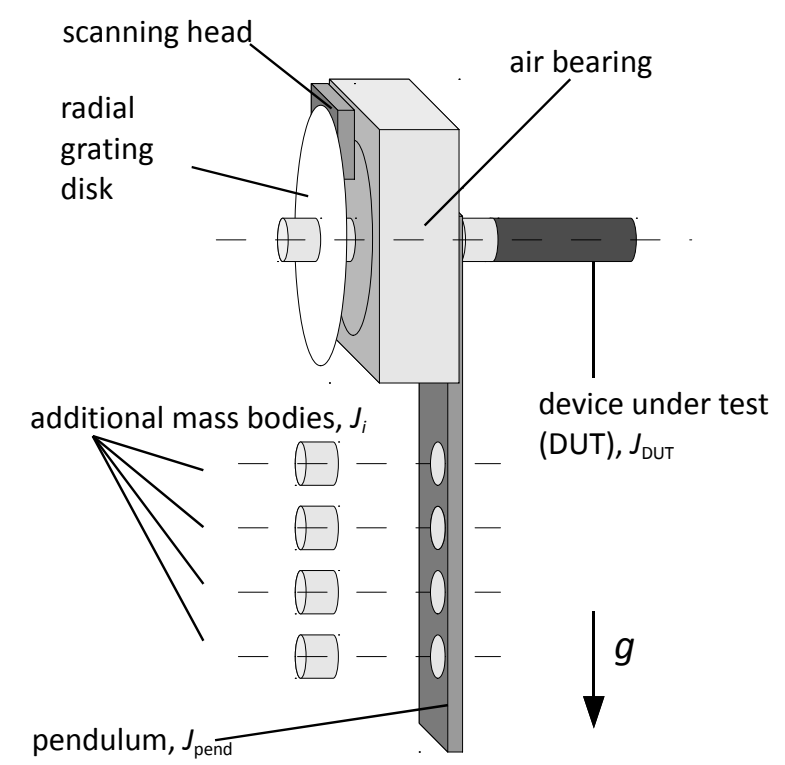

Figure 2. Pendulum for the measurement of the mass moment of inertia. 


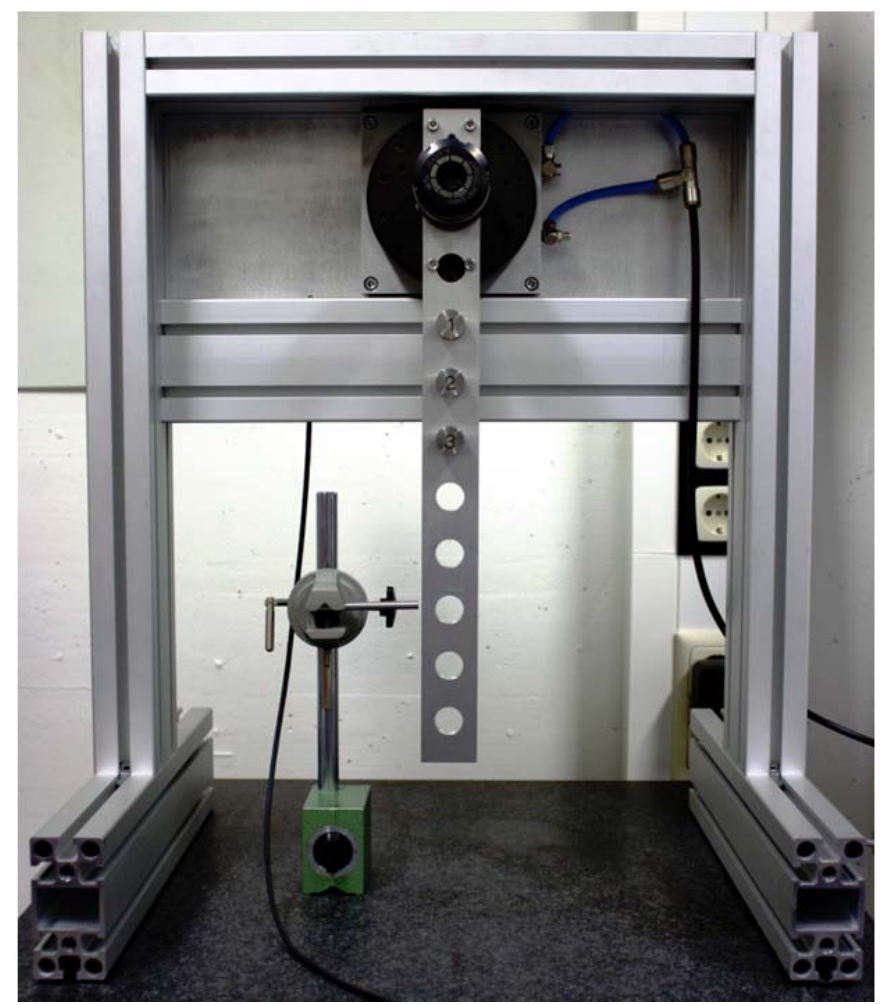

Figure 3. Measurement set-up for the determination of the mass moment of inertia, shown with three mounted mass bodies and mounted DUT.

angular resolution can be additionally increased by interpolation methods.

The measured total mass moment of inertia $J_{\text {total }}$ is composed of the MMOI of the pendulum $J_{\text {pend }}$ and the MMOI of all mounted components, e.g. of the device under test $J_{\text {DUT }}$ and of the added mass bodies $J_{i}$ of each test configuration $i$.

$J_{\text {total }}=J_{\text {DUT }}+J_{\text {pend }}+\sum J_{i}=J_{0}+\sum J_{i}$

As the pendulum's mass moment of inertia $J_{\text {pend }}$ is unknown, it has to be determined first. This is to be carried out in the same procedure as used subsequently for the mounted device under test. The unknown property can be identified by adding known mass moments of inertia to the pendulum. This is measured by mounting auxiliary mass bodies at different distances from the axis of rotation into the pendulum lever.

The mass bodies of the pendulum have well-known dimensions and weights. With this information, the mass moment of inertia for rotation around the centre of gravity of each mass body can be calculated. The distance of the mounting position of the masses to the rotational axis of the pendulum was measured in advance. The acting mass moment of inertia $J_{i}$ of each mass body at a certain mounting position is given by the Huygens-Steiner theorem.

Mounted mass bodies do not only influence the total mass moment of inertia of the pendulum, but also its total mass and its centre of gravity, i.e. the restoring torque $(m \cdot g \cdot l)$ on the right-hand side of Equation (2) is influenced. Each test configuration is described by
$J_{0}+\sum J_{i}=\frac{\left(m_{0} \cdot l_{0} \cdot g\right)+\sum\left(m_{i} \cdot l_{i} \cdot g\right)}{\omega_{i}^{2}}$

To determine the swing frequency of the pendulum in a given test configuration, the angle encoder output is sampled with a sufficiently high sampling rate for a similar range of pendulum excitation angles. After the predetermination of frequency $\omega$, magnitude $C$, phase $\varphi$ and decay-rate $\delta$, a four parameter Levenberg-Marquardt fit is calculated on a damped sine function

$$
y(t)=C \cdot e^{-\delta t} \cdot \sin (\omega t+\varphi)
$$

where the predetermined values are used as initial parameters. Experience gathered after the first measurements shows a stable behaviour of the fit and a very good agreement of measured data and fit.

The unknown mass moment of inertia $J_{0}$ can be derived by varying the configuration of the pendulum by means of mounting different auxiliary mass bodies. Several measurements are necessary to vary the two parameters mass moment of inertia and restoring torque sufficiently for the identification process.

This leads to a solvable bivariate regression problem in the form of

$y=f(X, p)$.

The vector of observed values $y$ consists of all of the measured angular frequency values $\omega_{i}$ of the corresponding configuration $i$; the added auxiliary mass bodies contribute to the matrix of independent values $X$, which consist of $\sum J_{i}$ and $\sum\left(m_{i} \cdot l_{i} \cdot g\right)$. The vector of approximated parameters $p$ consists of the unknown mass moment of inertia of the pendulum $J_{0}$ and the unknown restoring torque $\left(m_{0} \cdot l_{0} \cdot g\right)$.

$\boldsymbol{y}^{T}=\left[\omega_{1}, \omega_{2}, \ldots, \omega_{n}\right]$

$$
\boldsymbol{X}=\left[\begin{array}{cc}
\sum J_{1} & \sum\left(m_{1} \cdot l_{1} \cdot g\right) \\
\sum J_{2} & \sum\left(m_{1} \cdot l_{2} \cdot g\right) \\
\vdots & \\
\sum J_{n} & \sum\left(m_{n} \cdot l_{n} \cdot g\right)
\end{array}\right]
$$

$\boldsymbol{p}^{\mathrm{T}}=\left[J_{0},\left(m_{0} \cdot l_{0} \cdot g\right)\right]$

For the successful identification of the unknown parameters it is beneficial to maximize the variation of the independent values. This can be achieved by choosing mass bodies and mounting positions in an optimized way. Figure 4 shows the influence of different mass body configurations on the squared pendulum swing time $T^{2}=4 \pi^{2} / \omega^{2}$ at the $z$-axis, and on the restoring torque $\sum\left(m_{i} \cdot l_{i} \cdot g\right)$ and the added mass moments of inertia $\sum J_{i}$ at the $x$ - and $y$-axis, respectively. 


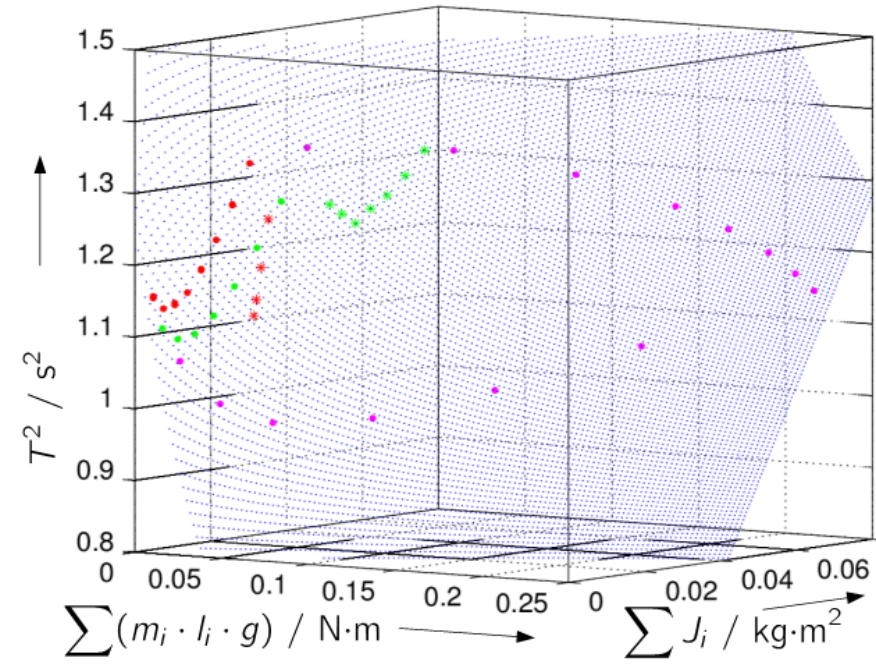

Figure 4. Measurement values for different auxiliary mass bodies mounted at different positions in the pendulum (red, green and magenta dots) and result of the bivariate regression (blue, dotted surface).

According to Equation 2, the pendulum is modelled assuming undamped oscillations. Therefore, this simplification can be applied only for systems of low damping. To estimate the effect of the damping, the decay of the pendulum's amplitude was measured (see Figure 5).

Differing from the previous description, the oscillations of the pendulum were measured by means of a laser-Doppler interferometer, as the angle measurement set-up had not yet been installed. The interferometer traced the pendulum's swing at the bottom end of the pendulum.

The damping of the pendulum was determined by calculating a non-linear regression approximating the measurement values with a damped sine fit function (see Equation (5)). The decay of the pendulum swing amplitude for the measurement data shown in Figure 5 was calculated and equals $\delta=2.23 \cdot 10^{-3} s^{-1}$. The damping slightly influences the resonant frequency of the pendulum [4]; the relationship between the damped natural frequency $\omega_{d}$ and the undamped frequency $\omega_{u d}$ is given by

$\omega_{\mathrm{ud}}=\sqrt{\left(\omega_{\mathrm{d}}^{2}+\delta^{2}\right)}$

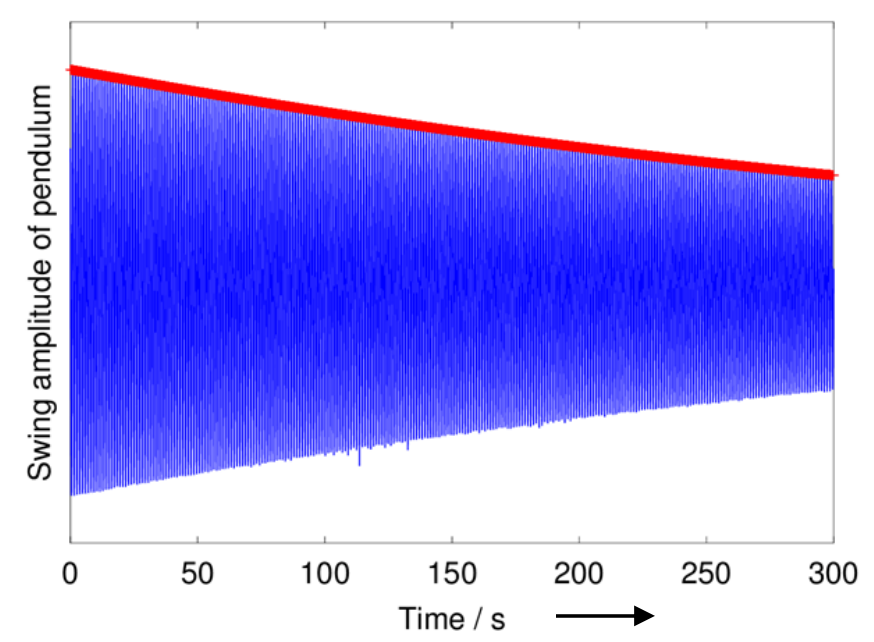

Figure 5. Decay of the pendulum's swing amplitude.

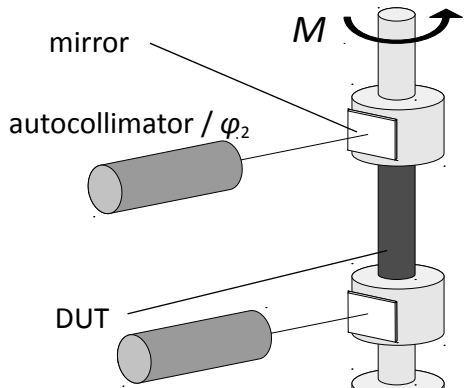

$\varphi_{1}$

reference torque transducer

\section{$-M \tau$}

Figure 6. Torsional stiffness measurement set-up.

For the measurement shown in Figure 5, the relative deviation due to this effect is in the range of $8 \cdot 10^{-8}$.

\section{TORSIONAL STIFFNESS}

The stiffness of the components of the measuring device has great influence on the torque measurement as it affects the dynamic behaviour of the whole system. The torsional stiffness $c$ is defined as the torque-to-torsion ratio (with torque $M$ and the torsional angle $\left.\Delta \varphi=\varphi_{2}-\varphi_{1}\right)$

$c=\frac{M}{\Delta \varphi}$

The determination of the torsional stiffness is realised by a measurement set-up (see Figure 6) in which a well-known torque is applied to the DUT while the angle of torsion is measured with high precision by means of two autocollimators.

Two mirrors attached with support clamps to both sides of the DUT provide the sensing surface of the autocollimators. The torque-dependent difference of the two angle readings of both autocollimators yields the torsional angle. Mirrors with a planarity of $1 / 10 \lambda$ enable the determination of the angle with uncertainties of less than one arcsecond; the measurement range amounts to rotational angles of up to \pm 600 arcseconds. In this measurement set-up, the statically applied torque is measured by means of a reference torque transducer.

To measure the torsional stiffness, PTB's $20 \mathrm{~N} \cdot \mathrm{m}$ Torque Calibration Machine was equipped with two electronic

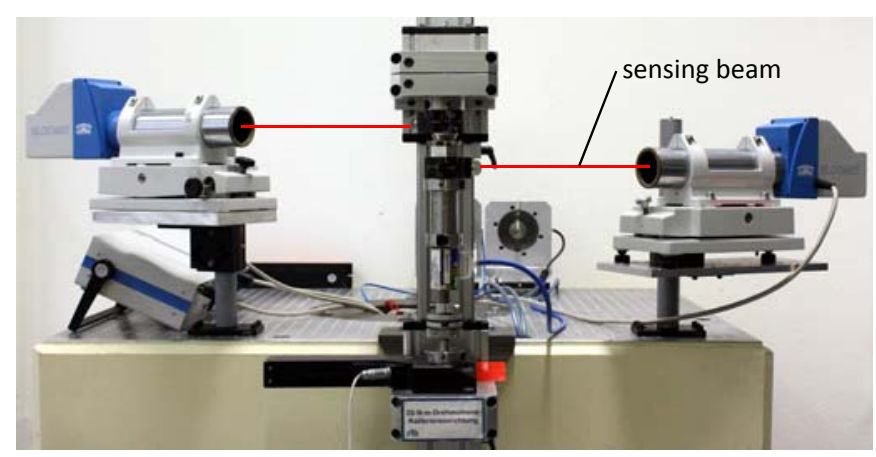

Figure 7. Measurement set-up for torsional stiffness with indicated sensing beams of autocollimators (red). 


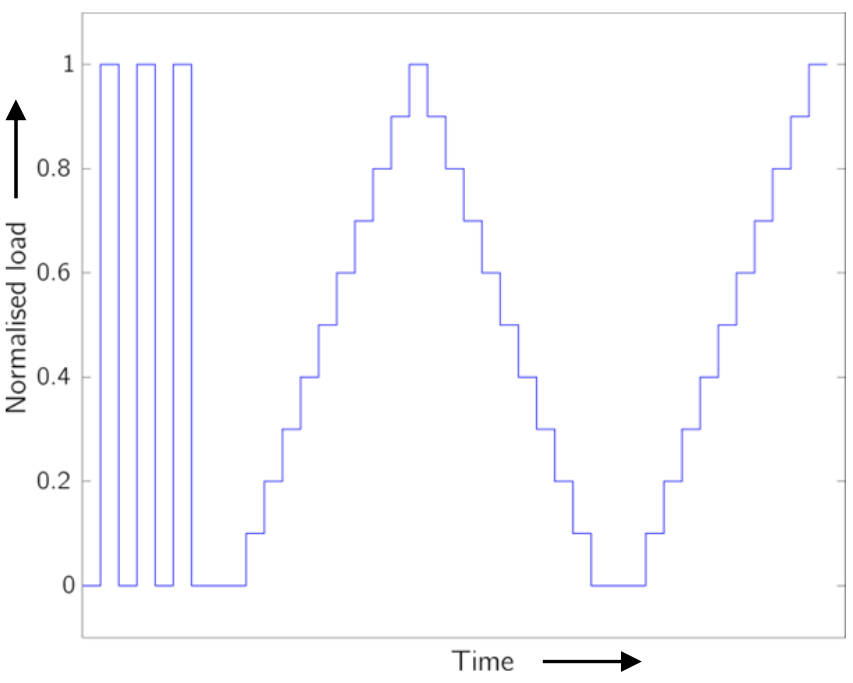

Figure 8. Load steps of the torsional stiffness measurement routine.

autocollimators in order to perform measurements of the torsional angle. Figure 7 shows a photograph of the set-up. The $20 \mathrm{~N} \cdot \mathrm{m}$ Torque Calibration Machine is seen in the centre, on the right is the autocollimator tracing the bottom mirror, on the left, the autocollimator sensing at the top mirror.

A measurement routine was developed for the evaluation of the torsional stiffness. The applied load steps are based on the DIN 51309 standard for torque transducer calibrations. After preloading to avoid hysteresis behaviour in the torque calibration system, the applied torque (load) increases in steps of $10 \%$ to the full load. The number of load steps shown in Figure 8 was increased in comparison to the standard. For the measurement, both a clockwise and a counter-clockwise torsional loading cycle were applied.

The voltage output of the bridge amplifier connected to the reference torque transducer and the angle values of the two autocollimators were acquired simultaneously. Several hundred measurement values were recorded for each torque step.

The first measurement results are very encouraging, as seen in Figure 9. The torsional angle values for the different torque steps show a linear dependency. The first order regression line excellently fits the mean values of the different torque levels. The value for torsional stiffness is given by the gradient of the regression line. The angle offset is given by the initial absolute angle values of the autocollimators.

\section{CONCLUSIONS}

This paper describes the modelling and the methods for the determination of the model parameters of the dynamic torque calibration device at PTB. This modelling of the measuring device is a prerequisite to be able to determine the transducer's dynamic properties.

The described methods and auxiliary measuring set-ups enable the measurement of the torsional stiffness and the mass moment of inertia. The torsional stiffness is determined by applying a well-known torque and by measuring the torsional angle on both sides of the DUT. The mass moment of inertia is determined by means of the swing frequency of a compound pendulum.

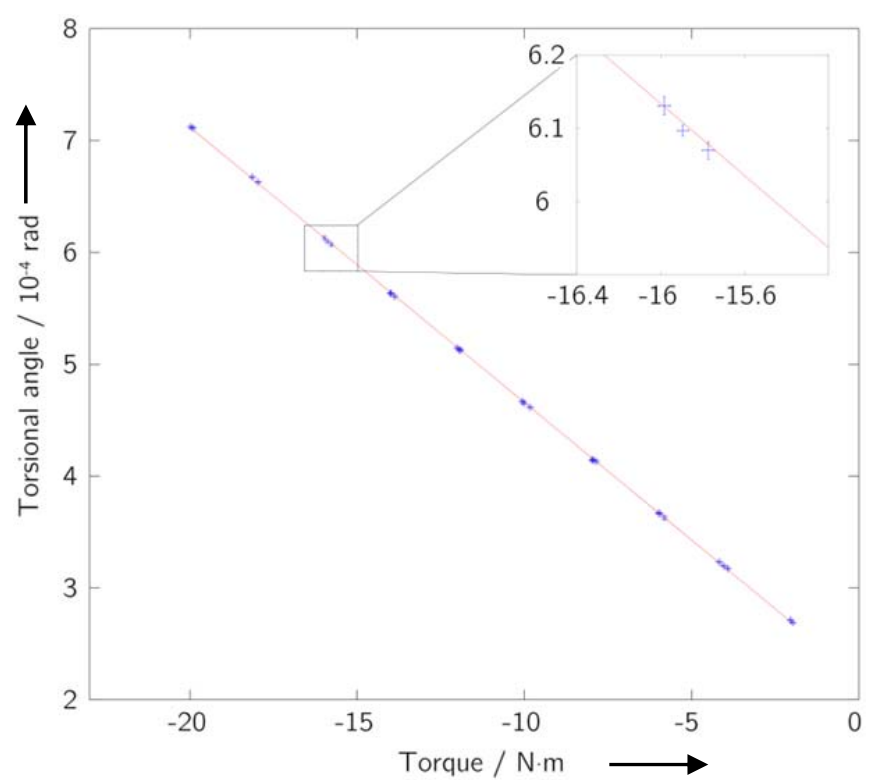

Figure 9. Measurement result with standard deviation (blue) and regression line (red).

As the commissioning of the described measuring set-ups is already completed, it is now intended to subsequently determine the model parameters of the components of the dynamic torque calibration device with these measurement methods.

For the future model-based calibration of torque transducers, the parameters of the measuring device need to be known in order to identify the model parameters of the torque transducer under test from measurement data.

\section{ACKNOWLEDGEMENT}

The authors would like to thank their colleagues Mr Brüge from the Working Group "Realization of Torque" for the opportunity to utilise the $20 \mathrm{~N} \cdot \mathrm{m}$ Torque Calibration Machine for the measurements of torsional stiffness, and Mr Just from the Working Group "Angle Metrology" for his helpful suggestions in angle measurement and for providing the autocollimators.

\section{REFERENCES}

[1] T. Bruns, "Sinusoidal Torque Calibration: A Design for Traceability in Dynamic Torque Calibration" in Proc. of XVII IMEKO World Congress; 2003, Dubrovnik, Croatia, CD publication, online at: http://www.imeko.org/publications/wc-2003/PWC-2003-TC3008.pdf.

[2] M. Kobusch, A. Link, A. Buss, T. Bruns, "Comparison of Shock and Sine Force Calibration Methods" in Proc. of IMEKO TC3 \& TC16 \& TC22 International Conference; 2007, Merida, Mexico, CD publication, online at: http://www.imeko.org/publications/tc3-2007/IMEKO-TC32007-007u.pdf.

[3] D. Peschel, D. Mauersberger, "Determination of the friction of aerostatic bearings for the lever-mass system of torque standard machines" in Proc. of XIII IMEKO World Congress, 1994, Torino, Italy, pp. 216-220.

[4] G. Baker, J. Blackburn, The pendulum: A case study in physics, Oxford University Press, Chapter 3, pp. 30-31, 2005. 